\title{
Beware of the dangers along the path towards the diagnosis of HPV-driven oropharyngeal squamous cell carcinoma
}

\author{
Attenzione alle insidie riservate dalla diagnosi del carcinoma squamocellulare \\ orofaringeo HPV-relato \\ P. BOSCOLO-RIZZO', F. BUSSU2 \\ ${ }^{1}$ Department of Neurosciences, Section of Otolaryngology, University of Padua, Treviso, Italy; ${ }^{2}$ Institute of \\ Otolaryngology, Università Cattolica del Sacro Cuore, Rome, Italy
}

Acta Otorhinolaryngol Ital 2017;37:63-64

Dear Editor,

A growing incidence of oropharyngeal squamous cell carcinoma (OPSCC) has been observed in many areas in high income countries. This so-called cancer epidemic has been attributed to an increasing causal role of highrisk alpha human papillomavirus (HR $\alpha$-HPV) in this malignancy. HR $\alpha$-HPV types, mainly HPV type 16 , are now ascertained to be aetiologically associated with a subset of OPSCC arising from the crypt epithelium of the palatine and lingual tonsils ${ }^{1}$. In addition, HR $\alpha$-HPVs are responsable for a substantial fraction of SCCs from unknown primary metastatic to the neck nodes (CUP) ${ }^{2}$.

Identifying those HR $\alpha$-HPV infections that are the real driving force behind the oropharyngeal carcinogenesis process is of paramount importance. Unlike cervical and other anogenital cancers, HR $\alpha$-HPVs play a causal role only in a subset of OPSCCs to which they confer a significantly better prognosis ${ }^{1}$. Furthermore, several ongoing clinical trials are exploring the feasability of treatment de-escalation in this subset of OPSCC in the attempt to reduce toxicity, while maintaining the same efficacy of traditional treatment regimens ${ }^{3}$.

Detection of HPV E6/E7 mRNA by polymerase chain reaction (PCR) in frozen samples is considered the gold standard for diagnosis of oncologically-relevant HPV infections. A spliced version of the E6 transcript, the ultra-short E6*I mRNA, can be successfully analysed even in formalin-fixed, paraffin-embedded (FFPE) specimens ${ }^{4}$. In a clinical setting, HPV-status is mainly assessed using FFPE-based diagnostic tests such as HR $\alpha$-HPV DNA detection (by PCR or in situ hybridisation) or immunostaining for cyclin-dependent kinase inhibitor p16 $6^{\mathrm{INK} 4 \mathrm{a}}$, considered a surrogate marker for active HPV involvement in OPSCC tumourigenesis. Unfortunately, both markers lack adequate sensitivity and/or specificity.
In particular, HR $\alpha$-HPV DNA testing positive to PCR sequencing may be linked to transient, non-transforming HPV infection or to specimen processing/laboratory contamination ${ }^{5}$. In addition, p16 ${ }^{\mathrm{INK} 4 \mathrm{a}}$ over-expression could be triggered by HPV-independent deregulation of $\mathrm{p} 16^{\mathrm{IN}-}$ ${ }^{\mathrm{K} 4 a} / \mathrm{RB}$ signalling pathway ${ }^{6}$. Finally, fluorescence in situ hybridisation, which is characterised by acceptable specificity, lacks sufficient sensitivity ${ }^{7}$.

Consistently, prognostic stratification based on HR $\alpha$-HPV-DNA or p16 $6^{\text {INK4a }}$ immunostaining as standalone tests is unsatisfactory compared to those based on more accurate markers of transforming infection such as E6/ E7 mRNA, high viral load, or E6 seropositivity ${ }^{8}{ }^{9}$. On the other hand, double positivity to HR $\alpha$-HPV-DNA and p16 $6^{\text {INK4a }}$ immunostaining is largely accepted to be a good compromise when oncologically-relevant infections are being determined in a clinical context ${ }^{10}$.

Surprisingly, most of the ongoing clinical trials exploring treatment de-escalation in patients with supposed HR $\alpha$-HPV-related OPSCC are recruiting patients on the basis of p16 ${ }^{\text {INK4a }}$ immunostaining positivity alone ${ }^{3}$, a modality that raises ethical concerns because it would expose a fraction of the patients whose $\mathrm{p} 16^{\mathrm{INK} 4 \mathrm{a}}$ over-expression is triggered by a HPV-independent mechanism to suboptimal treatment. In addition, translating the results of those studies into clinical practice/decision-making in areas where there is a low prevalence of HR $\alpha$-HPV-driven OPSCC could prove to be even riskier given the modest predictive value of $\mathrm{p} 16^{\mathrm{INK4a}}$ positivity.

Given these considerations, we strongly recommend aiming for at least positivity to both HR $\alpha$-HPV-DNA and p16 $6^{\text {INK4a }}$ immunostaining to estimate the burden of HR $\alpha$-HPV-driven OPSCC and to obtain more accurate predictive and prognostic information. p16 $6^{\mathrm{INK} 4 \mathrm{a}}$ immunostaining can be used as screening test and followed by HR $\alpha$-HPV-DNA testing on $\mathrm{p} 16^{\mathrm{INK} 4 \mathrm{a}}$-positive cases. We also 
recommend to routinely assess HPV-status in patients with CUP, as it may guide successful identification of the primary tumour in the oropharynx, provide important prognostic information and help to prevent comprehensive prophylactic mucosal irradiation of the entire upper aero-digestive tract.

\section{References}

1 Boscolo-Rizzo P, Mistro AD, Bussu F, et al. New insights into human papillomavirus-associated head and neck squamous cell carcinoma. Acta Otorhinolaryngol Ital 2013;33:77-87.

2 Bussu F, Sali M, Gallus R, et al. HPV and EBV infections in neck metastases from occult primary squamous cell carcinoma: another virus-related neoplastic disease in the head and neck region. Ann Surg Oncol 2015;22(Suppl 3):S979-984.

3 Mirghani H, Amen F, Blanchard P, et al. Treatment de-escalation in HPV-positive oropharyngeal carcinoma. Ongoing trials, Crit Issues Perspect. Int J Cancer 2015;136:1494-503.

4 Halec G, Schmitt M, Dondog B, et al. Biological activity of probable/possible high-risk human papillomavirus types in cervical cancer. Int J Cancer 2013;132:63-71.
5 Boscolo-Rizzo P, Pawlita M, Holzinger D. From HPV-positive towards HPV-driven oropharyngeal squamous cell carcinomas. Cancer Treat Rev 2016;42:24-9.

6 Rietbergen MM, Snijders PJ, Beekzada D, et al. Molecular characterization of p16-immunopositive but HPV DNA-negative oropharyngeal carcinomas. Int J Cancer 2014;134:2366-72.

7 Schache AG, Liloglou T, Risk JM, et al. Evaluation of human papilloma virus diagnostic testing in oropharyngeal squamous cell carcinoma: sensitivity, specificity, and prognostic discrimination. Clin Cancer Res 2011;17:6262-71.

8 Holzinger D, Schmitt M, Dyckhoff G, et al. Viral RNA patterns and high viral load reliably define oropharynx carcinomas with active HPV16 involvement. Cancer Res 2012;72:4993-5003.

9 Liang C, Marsit CJ, McClean MD, et al. Biomarkers of HPV in head and neck squamous cell carcinoma. Cancer Res 2012;72:5004-13.

10 Rietbergen MM, Leemans CR, Bloemena E, et al. Increasing prevalence rates of HPV attributable oropharyngeal squamous cell carcinomas in the Netherlands as assessed by a validated test algorithm. Int J Cancer 2013;132:1565-71. 